\title{
Ancient recombination events and the origins of hepatitis E virus
}

\author{
Andrew G. Kelly, Natalie E. Netzler and Peter A. White
}

\begin{abstract}
Background: Hepatitis E virus (HEV) is an enteric, single-stranded, positive sense RNA virus and a significant etiological agent of hepatitis, causing sporadic infections and outbreaks globally. Tracing the evolutionary ancestry of HEV has proved difficult since its identification in 1992, it has been reclassified several times, and confusion remains surrounding its origins and ancestry.

Results: To reveal close protein relatives of the Hepeviridae family, similarity searching of the GenBank database was carried out using a complete Orthohepevirus A, HEV genotype I (GI) ORF1 protein sequence and individual proteins. The closest non-Hepeviridae homologues to the HEV ORF1 encoded polyprotein were found to be those from the lepidopteran-infecting Alphatetraviridae family members. A consistent relationship to this was found using a phylogenetic approach; the Hepeviridae RdRp clustered with those of the Alphatetraviridae and Benyviridae families. This puts the Hepeviridae ORF1 region within the "Alpha-like" super-group of viruses. In marked contrast, the HEV GI capsid was found to be most closely related to the chicken astrovirus capsid, with phylogenetic trees clustering the Hepeviridae capsid together with those from the Astroviridae family, and surprisingly within the "Picorna-like" supergroup. These results indicate an ancient recombination event has occurred at the junction of the non-structural and structure encoding regions, which led to the emergence of the entire Hepeviridae family. The Astroviridae capsid is also closely related to the Tymoviridae family of monopartite, $T=3$ icosahedral plant viruses, whilst its non-structural region is related to viruses of the Potyviridae; a large family of plant-infecting viruses with a flexible filamentous rod-shaped virion. Thus, we identified a separate inter-viral family recombination event, again at the non-structural/structural junction, which likely led to the creation of the Astroviridae.

Conclusions: In summary, we have shown that new viral families have been created though recombination at the junction of the genome that encodes non-structural and structural proteins, and such recombination events are implicated in the genesis of important human pathogens; HEV, astrovirus and rubella virus.
\end{abstract}

Keywords: Hepatitis E virus, Hepatitis, Recombination, Hepeviridae, Astroviridae, Evolution

\section{Background}

Hepatitis E virus (HEV) is a single-stranded RNA, nonenveloped enteric virus and a major etiological agent responsible for acute hepatitis, causing sporadic infections and widespread epidemics globally. Based on seroprevalence, it is estimated that one third of the global population has been infected with HEV at some stage in their lifetime [1]. HEV creates a significant health burden on society, with annual estimates of 20 million clinical infections, over three million reported acute cases, 3,000 stillbirths and 50,000 HEV-related deaths globally [2, 3].

\footnotetext{
* Correspondence: p.white@unsw.edu.au

School of Biotechnology and Biomolecular Sciences, Faculty of Science, University of New South Wales, Sydney, NSW, Australia
}

However, it is likely that these figures are an underestimation, given that the virus is often asymptomatic, usually self-limiting and HEV epidemics strike in geographic areas that are the least likely to have effective diagnostic testing available $[4,5]$.

The Hepeviridae family is currently divided into two genera, Orthohepevirus and Piscihepevirus [6]. These genera include the species: Orthohepevirus A, which infects humans, swine, boar, rabbits, camels, deer and mongooses, Orthohepevirus B, which infects birds, Orthohepevirus $\mathrm{C}$, which infects rodents and ferrets and Orthohepevirus D, which infects bats, whilst Piscihepevirus includes cutthroat trout virus (CTV) [6]. There are currently seven recognized Orthohepevirus A genotypes. 
Genotype (G) I and GII only infect humans, while GIII and GIV infect a range of animal hosts, as well as humans. GV and GVI infect boars, while GVII infects camelids and humans. Orthohepevirus B has four proposed genotypes, Orthohepevirus $\mathrm{C}$ includes two genotypes (HEV-C1 and HEV-C2), while Orthohepevirus D has a single genotype [6]. Additionally there is an unclassified moose HEV, which was recently identified in Sweden [7].

\section{HEV classification}

An initial analysis of the HEV GI non-structural polyprotein in 1992 [8] indicated that the polymerase and helicase sequences were closely related to a plant virus, beet necrotic yellowing vein virus (BNYVV). BNYVV is a member of the newly proposed family Benyviridae and is a causative agent of rhizomania in sugar beet. This virus was first reported in Italy in 1966 [9], and was subsequently isolated in Japan in 1973 [10], and found to be transmitted by an obligate intracellular parasite protist vector, Polymyxa betae [11]. HEV and BNYVV both have positive sense single-stranded RNA (ssRNA (+)) genomes, although that of BNYVV is segmented into 4-5 separate RNA fragments [12], compared to the monopartite genome of HEV. Comparative analysis of the HEV RNA-dependent RNA polymerase (RdRp), methyltransferase and helicase protein sequences with other ssRNA (+) viral sequences also revealed a genetic relationship with rubella virus (RuBV) $[8,9]$. These findings led to the initial inclusion of HEV within the "alpha-like" supergroup of viruses, based on similarities in their RdRp, helicase and methyltransferase amino acid sequences and also the presence of a putative papain-like cysteine protease $[8,13]$.

A comparison of the genomic organization of $\mathrm{HEV}$ proteins with that of other viruses, and the absence of glycoproteins, led to an entirely new suggestion that HEV might have evolved from an ancestral calicivirus [8]. Surprisingly, this led to the inclusion of HEV within the Caliciviridae family, even though the Caliciviridae were already placed in the "Picorna-like" supergroup, and quite different to the "alpha-like" supergroup of viruses $[8,14,15]$ (Fig. 1). Ultimately this led to some confusion over the ancestry of HEV, which was partially resolved in 1997, when the Caliciviridae were then subsequently organized into five genogroups, annexing HEV into a distinct sub-family by itself, but still within the Caliciviridae [16] (Fig. 1).

In 2000, phylogenetic analyses using more sequence data (including four different HEV strains), again analyzing capsid, helicase and RdRp conserved regions, moved HEV out of the Caliciviridae and into a separate, unassigned clade of indeterminate taxonomic position, but closer to the Togaviridae (which includes RuBV) [17], within the "alpha-like" supergroup. This finally resolved the problem of HEV being both a member of the "Picorna-like" supergroup and the "alpha-like" supergroup. As more and more sequences became available, HEV was seen to be more and more distinct from other viral genera and families, and it was accorded its own genus (Hepevirus) by 2004 and then its own family (Hepeviridae) in 2009 [18, 19]. In 2014, the taxonomy of the Hepeviridae was reviewed again and the family was divided into two new genera: Orthohepevirus and Piscihepevirus, as discussed earlier [6] and in 2016, Smith and colleagues proposed reference sequences for HEV subtypes within the Orthohepevirus genus [20]. Figure 1 illustrates the timeline of HEV classification through the International Committee on Taxonomy of Viruses (ICTV).

Despite scrutiny of the non-structural protein encoding region, the origin of the $\mathrm{HEV}$ capsid remained unresolved, since the Benyviridae are non-enveloped rod-shaped plant viruses [12], whereas HEV capsids are $\mathrm{T}=3$ icosahedral in shape, with an estimated 180 copies of the capsid protein $[21,22]$. It was not until 2011, that surprisingly the HEV capsid protein was shown to be most structurally related to capsids from members of the Astroviridae family of vertebrate-infecting viruses [23]. Astroviruses, like $\mathrm{HEV}$, also have a $\mathrm{T}=3$ icosahedral capsid, but which belong with the "Picorna-like" supergroup of viruses. To this day confusion over the ancestry of HEV is still apparent, as the non-structural protein-encoding region is classified within the "alphalike" supergroup, in contrast to the structural region. Therefore, this study aimed to shed further insight into the evolution and origins of the Hepeviridae family in its entirety. We initially undertook both a phylogenetic approach and an updated analysis of the identity between the individual HEV proteins and other viral proteins. Here we provide evidence that the whole Hepeviridae family likely arose through an ancient recombination event, which could have occurred between plant and insect viruses, with the breakpoint at the junction that separates the non-structural from the structural encoding regions of the genome.

\section{Results \\ Non-structural proteins \\ Hepeviridae evolution - Homology searches using the Orthohepevirus A HEV GI ORF1 polyprotein}

To reveal close protein relatives within the Hepeviridae family, similarity searching of the GenBank database using the complete Orthohepevirus A HEV GI ORF1 polyprotein sequence (Figs. 2 and 3) (accession number NP056779) was initially undertaken. The closest nonhuman Hepeviridae ORF1-encoded homologues to the human HEV GI ORF1-encoded polyprotein, were the ferret Orthohepevirus C polyprotein (BAO57189; $57 \%$ average amino acid identity over 1437 amino acids, in 


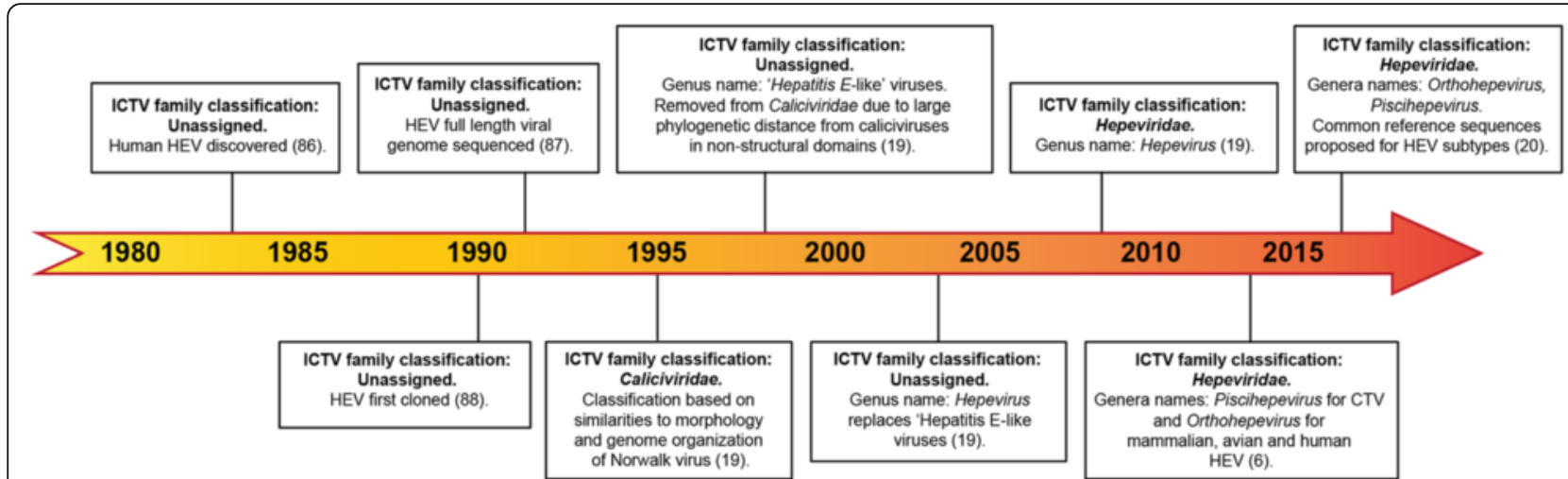

Fig. 1 Hepeviridae classification timeline. The changing classification of Hepeviridae over time as determined by the International Committee on Taxonomy of Viruses (ICTV), with relevant works cited [6, 19, 20, 86-88]

two fragments), followed by an avian Orthohepevirus B polyprotein (YP009001465; $49 \%$ average amino acid identity over 1442 amino acids, in two fragments), bat Orthohepevirus D polyprotein (YP006576507; 49 \% average amino acid identity over 1442 amino acids, in two fragments) and Piscihepevirus CTV polyprotein (YP004464917; 29 \% average amino acid identity over 1434 amino acids, in two fragments) (Fig. 3, Table 1). Surprisingly, the searches using the HEV GI ORF1 polyprotein also highlighted a unique 275 amino acid region of the HEV genome (between amino acids 498-773), comprising the putative papain-like protease sequence (NP056782), the polyproline region (NP056783) and 119 residues of intervening sequence (here termed the $\mathrm{Z}$ region) (Fig. 2). This unique 275 amino acid region within ORF1 appeared to have little or no homology to any non-Orthohepevirus A sequence (Fig. 3 and Additional file 1: Figure S1).

The closest non-Hepeviridae homologues to the Orthohepevirus A HEV GI ORF1 polyprotein were found to be from lepidopteran-infecting Alphatetraviridae family members (Fig. 3). Of the Alphatetraviridae, the highest scoring ORF1-encoded homologue by tBlastn or Blastp searching was Helicoverpa armigera (cotton bollworm) stunt virus (HASV) (genus Omegatetravirus), with homology to the MTase and helicase/RdRp regions (EU345431: $26 \%$ and $29 \%$ identity from two fragments comprising 823 amino acids; E-values $=1.74 \times 10^{-26}$ and
$2.03 \times 10^{-07}$ ) (Fig. 3 and Table 1). Other Alphatetraviridae viruses with homology to the HEV ORF1 included Dendrolimus punctatus tetravirus (AY594352: $25 \%, 25 \%$ and $20 \%$ identity from three fragments comprising 1,116 amino acids, E-values $=1.28 \times 10^{-21}$ and $7.42 \times 10^{-8}$ ), and Nudaurelia capensis beta virus (NP048059: $25 \%$ and $20 \%$ identity from two fragments comprising 1,104 amino acids) (Fig. 3).

Other noteworthy homologies were found, in the RdRp region, with a Sclerotinia sclerotiorum RNA virus [24], an unassigned dsRNA mycovirus infecting the plant pathogenic fungus S. sclerotiorum, (EU779934: $28 \%$ average identity over 218 amino acids; E-value $=1.17 \times 10^{-9}$ ); an unclassified tick-borne tetravirus-like virus [25] isolated from the ixodid hard tick Dermacentor variabilis (KM048322: $25 \%$ average identity over 348 amino acids; E-value $=3.03 \times 10^{-17}$ ) and the unclassified Chara australis virus [26], an ssRNA(+) virus infecting the charophyte algae C. australis var. nobilis (JF824737: $30 \%$ average identity over 212 amino acids of the $\mathrm{RdRp}$ region; E-value $=4.73 \times 10^{-12}$ ) (Fig. 3). The previously noted similarities between the HEV RdRp region and Benyviridae and Togaviridae viruses [8] were also observed in this study (Fig. 3).

Away from the Alphatetraviridae family, numerous homologues $\left(1.0 \times 10^{-9}>\right.$ E-values $\left.>1.0 \times 10^{-12}\right)$ were found within the Betaflexiviridae family of plant viruses

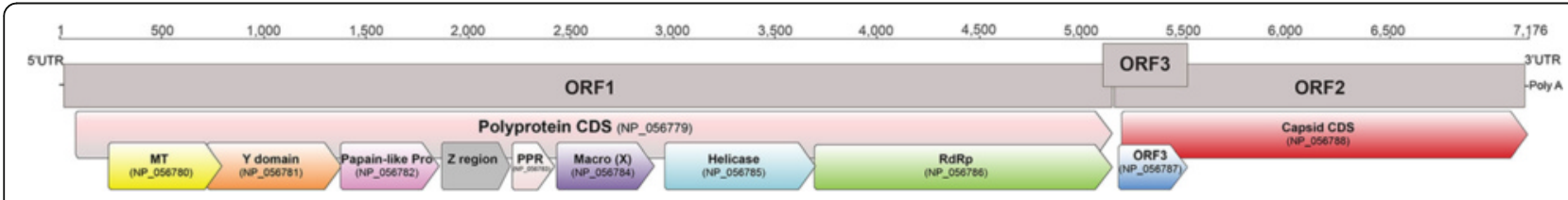

Fig. 2 HEV GI protein organization of the encoded genome. The complete HEV GI prototype genome (GenBank accession number NC_001434), showing the individual ORFs 1 to 3 and their encoded proteins. The numbers along the top indicate nucleotide positions. Protein domains within the ORF1 polyprotein are shown along the bottom. The ORF1 region encoded between nucleotides 1780 to 2136 inclusive (termed region Z) has not been ascribed to any specific protein. The $3^{\prime}$ and $5^{\prime}$ untranslated regions and the poly A tail are indicated 


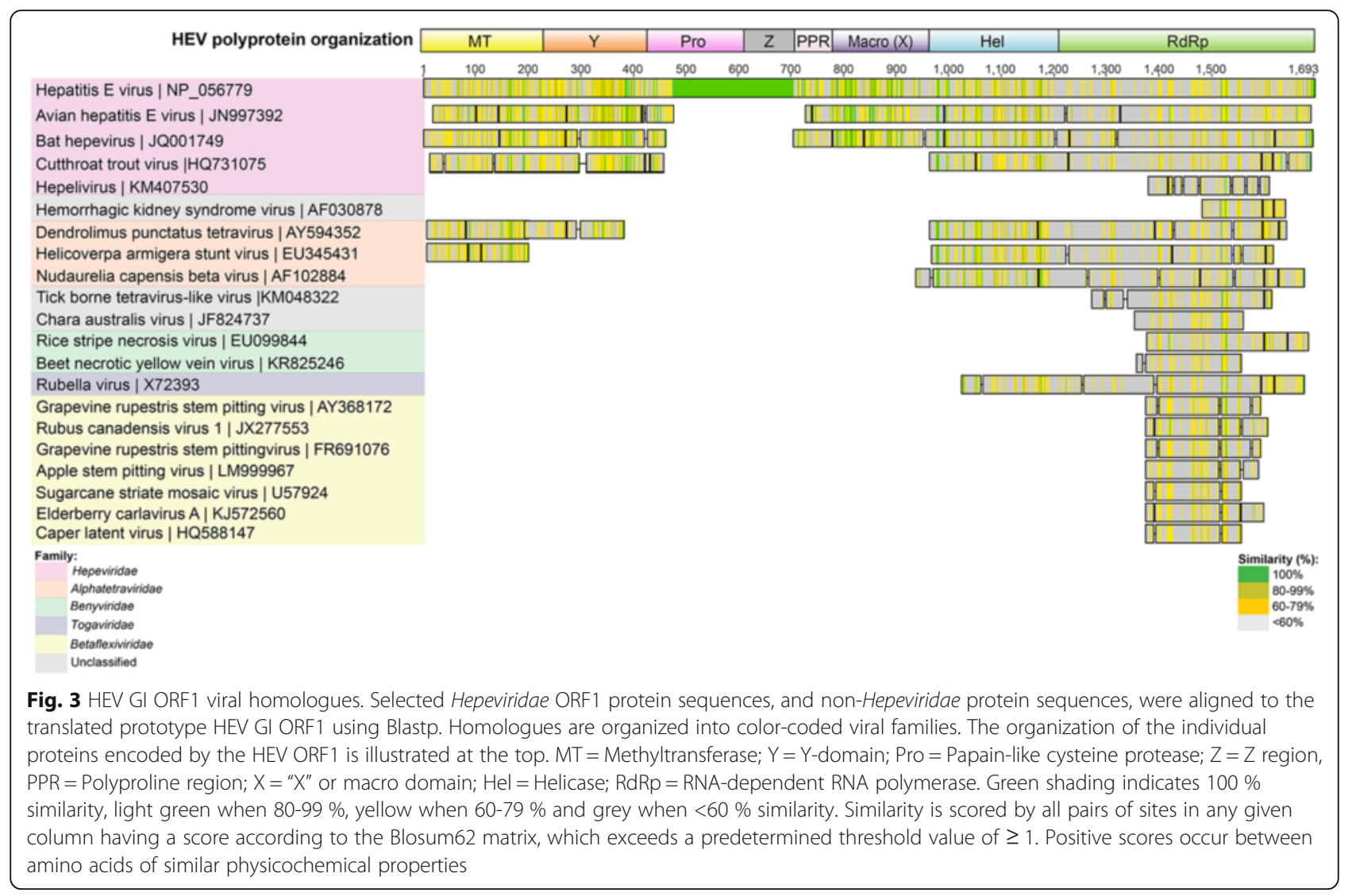

(Foveavirus, Carlavirus) and Virgaviridae family of plant viruses (Tobamovirus), primarily with the helicase and RdRp regions of human GI HEV (Figs. 3, 5 and 6).

\section{Hepeviridae evolution - Homology searches using the Orthohepevirus A HEV GI individual protein sequences} The genomic organization of the HEV ORFs 1, 2 and 3, as illustrated in Fig. 2, comprises the following nine proteins in order from left to right, starting from the $\mathrm{N}$-terminal: methyltransferase, Y-domain, papain-like cysteine protease, polyproline region, macro domain, helicase and RNA-dependent RNA polymerase (RdRp) from the polyprotein of ORF1, the capsid protein (ORF2) and phosphoprotein (ORF3). Additional tBlastn searches were undertaken using the individual Orthohepevirus A HEV GI protein sequences (accession numbers NP_056780 to NP_056788 inclusive) to identify any other, more distantly related viral protein homologues encoded by these regions. In particular, we wanted to determine whether the 275 amino acid region of the HEV GI genome between amino acids 498-773, described above, comprising the papain-like protease sequence, the polyproline region and $\mathrm{Z}$ region (the intervening sequence), had any non-Hepeviridae viral homologues, since it was poorly conserved within the Hepeviridae (Fig. 3 and Additional file 1: Figure S1). This search revealed a number of previously unidentified homologies to proteins from several viral families other than those previously recorded (such as the Alphatetraviridae, Betaflexiviridae, Closteroviridae, Mesoniviridae and Virgaviridae families; Figs. 3, 4, 5, 6 and 7) [8]. The individual protein homology searches are described below and the best hits are summarized in Table 1.

\section{Methyltransferase}

Out of the Hepeviridae, the closest homologous sequence to the Orthohepevirus A HEV GI methyltransferase protein (MTase; accession number NP056780) was the Orthohepevirus C ferret HEV methyltransferase region (accession number BAO57185; E-value $=5.47 \times 10^{-65}$, with an overall amino acid identity of $59 \%$ over 182 amino acids) (Table 1). The closest non-Hepeviridae homologue from a tBLASTn search was found to be a methyltransferase protein from the aforementioned HASV (E-value $=$ $5.88 \times 10^{-7}$ ) (Fig. 4, Table 1); a 150 amino acid region with $33 \%$ identity. Other members of the family such as Nudaurelia capensis beta virus (NBetaV, AF102884) were also observed to have weaker homology to the HEV MTase (Fig. 4). Representatives of the Tobamovirus genera (plant virus family Virgaviridae) were also weakly homologous to the HEV MTase $\left(10^{-6}<\right.$ E-value $\left.<10^{-4}\right)$. 


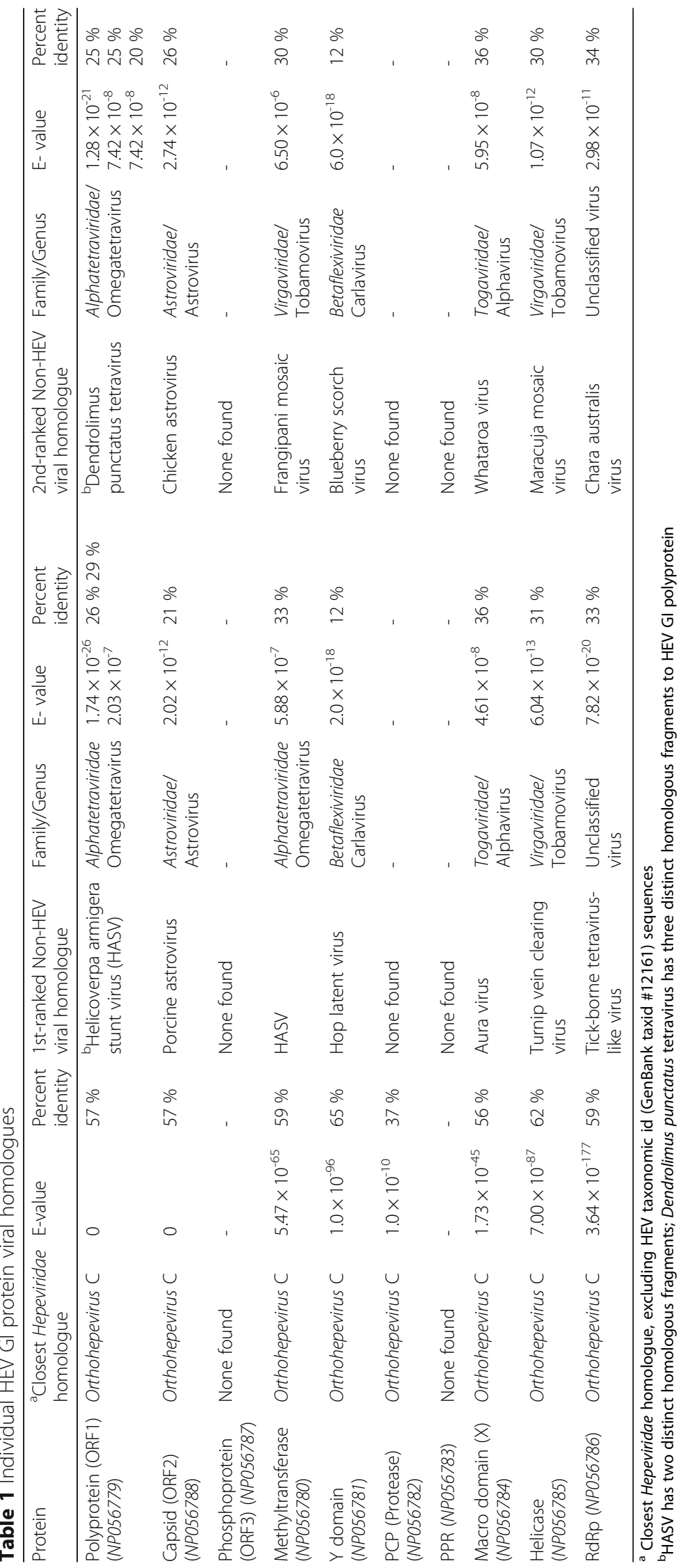




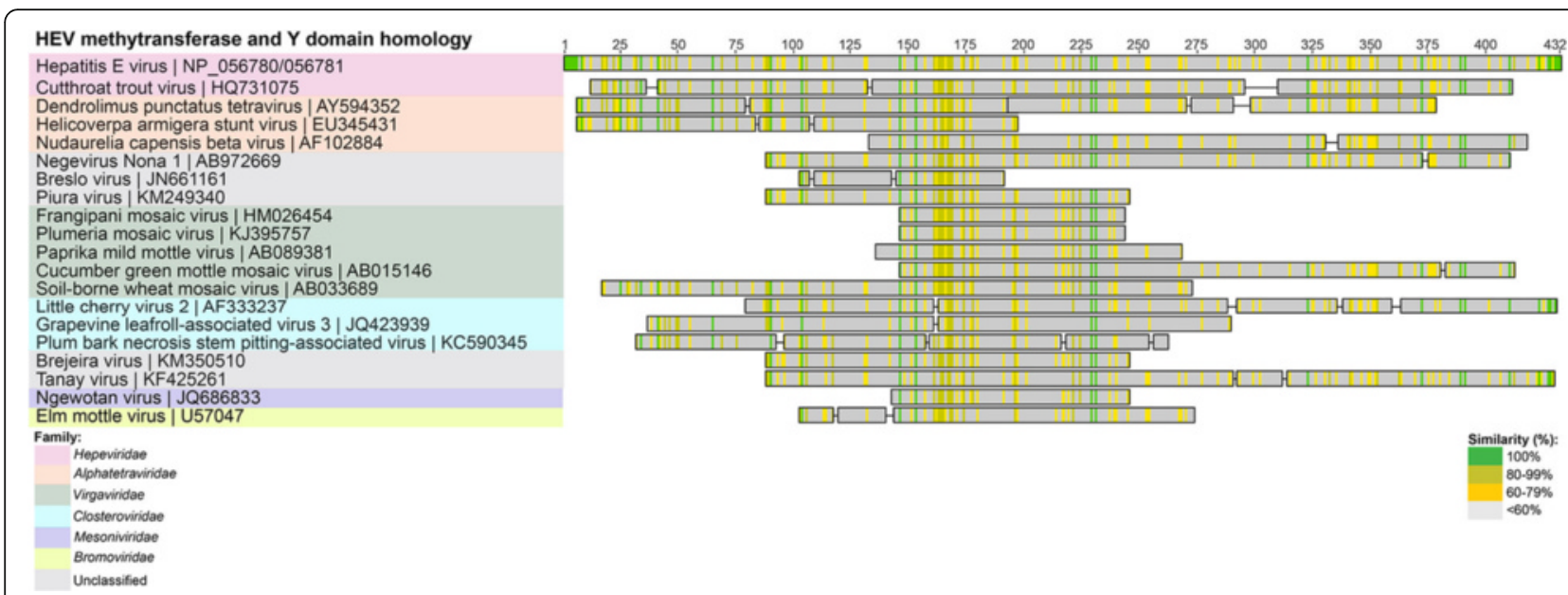

Fig. 4 HEV Gl Methyltransferase and Y domain viral homologues. Selected homologous sequences to concatenated HEV methyltransferase and Y-domain proteins identified using tBlastn search. Homologues are organized into color-coded viral families, as indicated in the legend. Shading as in Fig. 3

\section{Y-domain}

The HEV Y domain (accession number NP056781), immediately upstream of the MTase region (Fig. 2) was first described by Koonin et al. [8], but has no described function. It was found to be well conserved within the Hepeviridae, and the nearest homologue was the corresponding ferret HEV Y domain of the Orthohepevirus C ferret HEV ORF1 polyprotein (BAO57189; E-value $=1.0 \times 10^{-96}, 65 \%$ identity over the whole 217 amino acids (Table 1). Outside of the Hepeviridae, no significant identity was observed with the $\mathrm{Y}$ domain or any other protein (data not shown) using Blastp or tBlastn searches. However, a Delta-Blast search using a Blosum45 substitution matrix identified a region within the methyltransferase domain of Hop latent virus (NP066258; genus Carlavirus, of the Betaflexiviridae family of plant viruses), as the closest match, with an Evalue $=2.0 \times 10^{-18}$, with $12 \%$ identity over 138 amino acids.
An InterProScan analysis of a concatenated methyltransferase and $\mathrm{Y}$ domain sequence showed that when combined, they contain a single region of 348 amino acids, corresponding to the Pfam member "Vmethyltransf" $\left(\mathrm{PF} 01660, \mathrm{E}\right.$-value $\left.=7.5 \times 10^{-18}\right)$, suggestive of a larger contiguous MTase sequence, than that which is represented by NP_056780 alone. Pfam is a protein family database, comprising over 13,000 curated protein families that share significant homology as detected by the profile hidden Markov model method in the HMMER3 suite of programs [27].

An additional tBlastn search of the GenBank nonredundant viral database was undertaken, this time using the MTase and Y-domain concatenated sequence (Fig. 4). This identified the same hits as for the individual MTase and Y-domain searches above. However, the concatenated sequence search resulted in similar E-values, but with
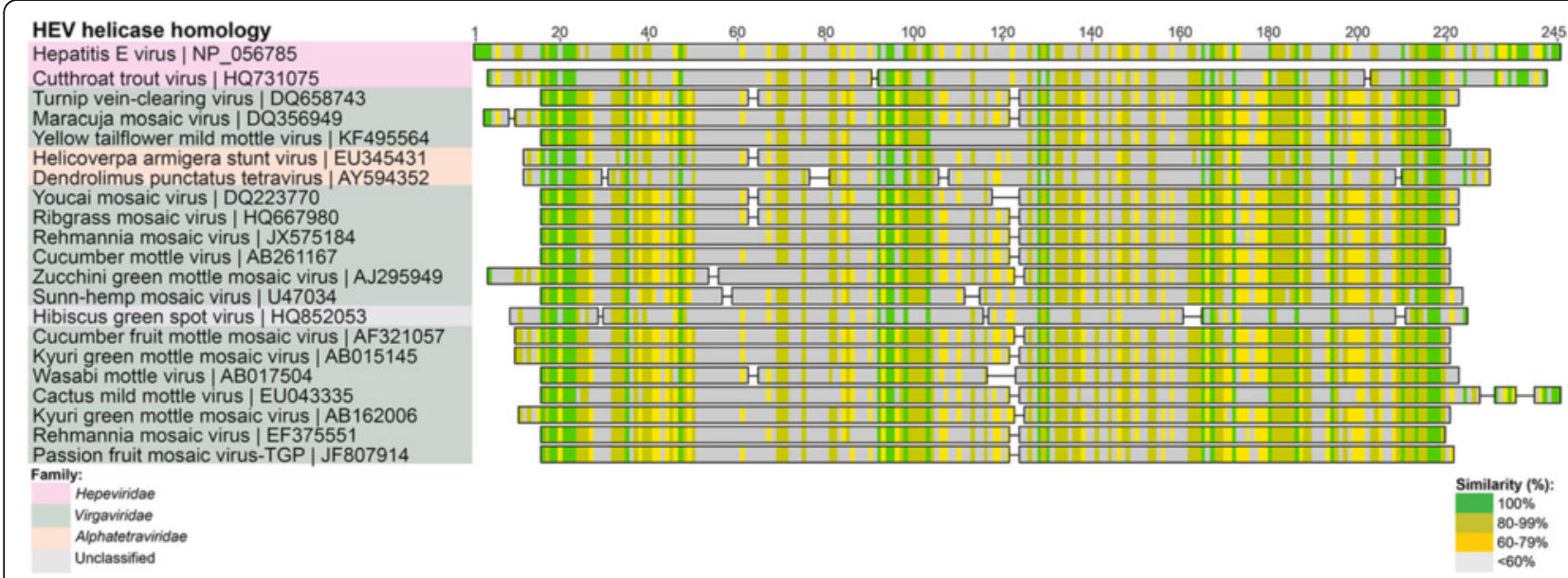

Fig. 5 HEV Gl Helicase viral homologues. Selected homologous sequences to concatenated HEV methyltransferase and Y-domain proteins identified using tBlastn search. Homologues are organized into color-coded viral families, as indicated in the legend. Shading as in Fig. 3 


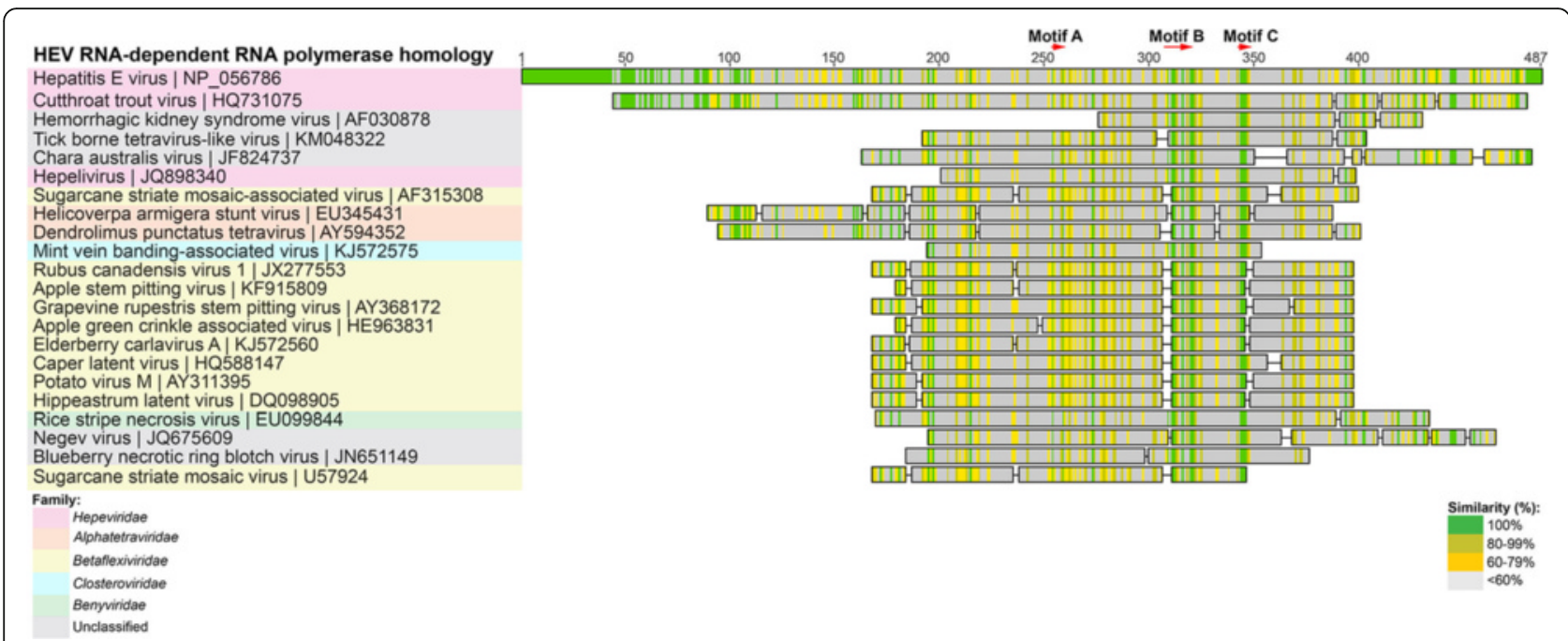

Fig. 6 HEV GI RdRp viral homologues. Selected homologous sequences to HEV RdRp identified using tBlastn search. Homologues are organized into color-coded viral families. Shading as in Fig. 3

extended regions of homology for some viruses, such as NBetaV (AF102884), which now had a region of 323 amino acids $\left(20 \%\right.$ identity, E-value $\left.=4.0 \times 10^{-5}\right)$, aligned with the concatenated sequences, compared to 146 amino acids for the MTase alone (24\% identity, E-value $=1.37 \times 10^{-5}$ ) (Fig. 4). In addition, with the extended sequence, new homologues were found with negeviruses, a recently described group of insect viruses [28]. The most homologous negeviral sequence was that of the polyprotein of Negevirus Nona 1, with $23 \%$ identity over 313 amino acids (AB972669, E-value $=1.95 \times 10^{-4}$ ); the homologous region corresponds to the putative methyltransferase domain [28] (Fig. 4).

\section{Papain-like cysteine protease}

The HEV papain-like cysteine protease domain, of 160 amino acids (PCP; accession number NP056782) is not very well conserved, even within the Hepeviridae, and the nearest non-human Hepeviridae homologue, rat Orthohepevirus C PCP, has homology only at the Nterminal region of the PCP, with $42 \%$ identity, from residues 33 to 78 . An alignment of this region with other

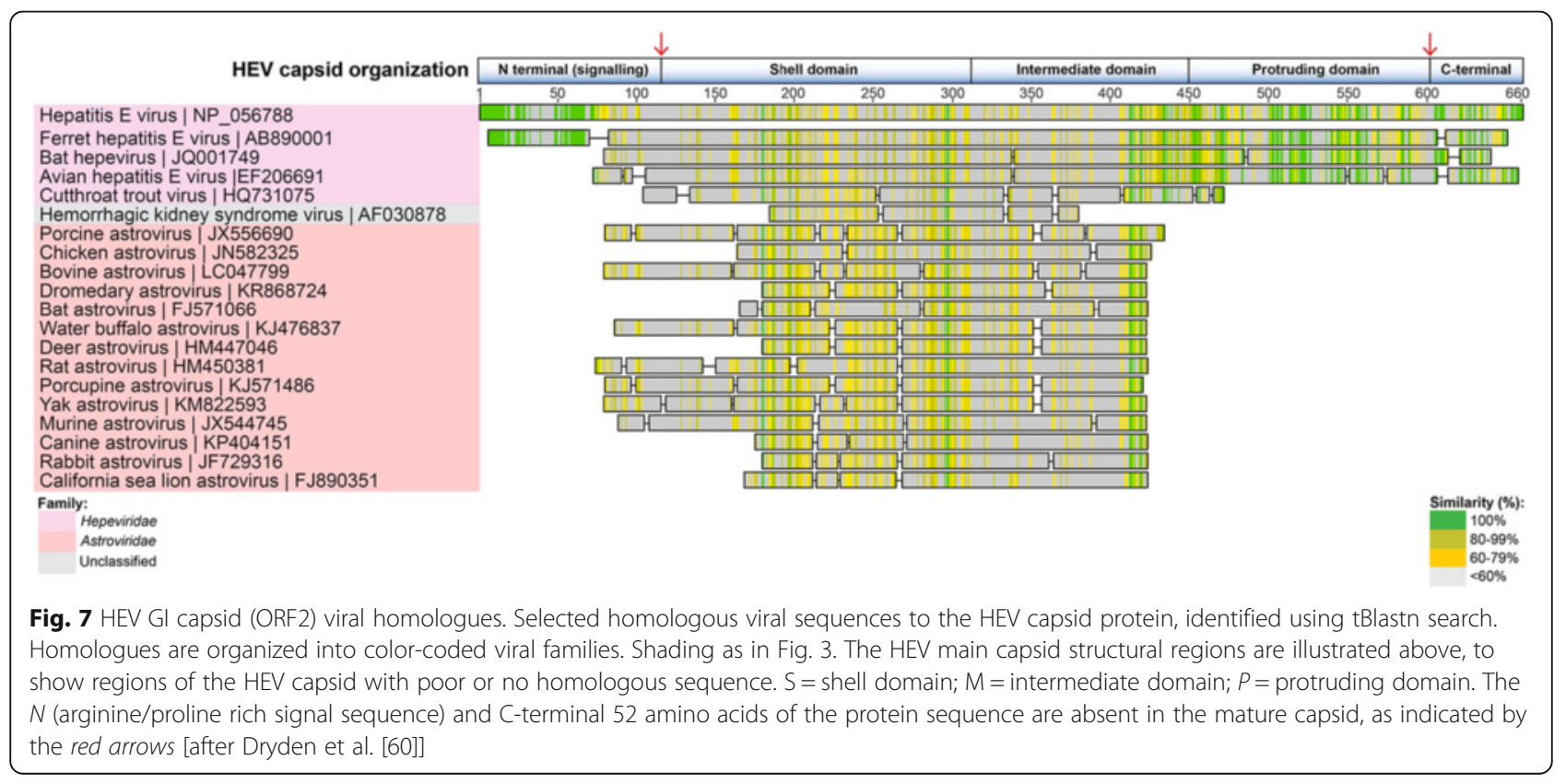


Hepeviridae family members illustrates the heterogeneity of this small region within the Hepeviridae, with low sequence identity across the family in this region (Additional file 1: Figure S1). Excluding the Hepeviridae from the search and using tBlastn, Blastp or PSI-Blast did not identify any significant homology with any other proteins in the GenBank database.

\section{Z Region}

The $\mathrm{Z}$ region comprises amino acids 593-711 inclusive, of the Orthohepevirus A HEV GI ORF1 polyprotein sequence (NP_056779). This region appears to be unique across the Orthohepevirus A, with amino acid sequence identity of $>68 \%$ over 119 residues for viruses within the species. However, region $\mathrm{Z}$ is highly variable in both sequence and length outside Orthohepevirus A. No significant similarity of the $\mathrm{Z}$ region was detected outside the Hepeviridae family.

\section{Polyproline region}

The polyproline region (PPR) of HEV, or hypervariable region (HVR) as it is also known (accession number NP056783), is an intrinsically disordered region (IDR), whose relaxed structure allows it to bind to multiple ligands, and be involved in the regulation of transcription and translation [29]. As its name suggests, the PPR contains a high level of proline residues, and is extremely variable in its sequence, even between Orthohepevirus A genotypes [30]. Despite significant inter-genotypic variation, within the Orthohepevirus A there is enough intragenotypic conservation in the PPR to allow the creation of genotype-specific phylogenetic trees (data not shown). Blastp, tBlastn and PSI-Blast searches did not identify any significant homologues with other non-human Hepeviridae viruses, nor any other sequence, viral or non-viral, outside of the Hepeviridae (data not shown).

\section{Macro (X) domain}

The macro domain (accession number NP056784) is involved in ADP-ribose metabolism and post-translational modifications. As well as being found in the Hepeviridae, homologues exist in the Coronaviridae and alphaviruses, as well as in bacteria and eukaryotes [31]. Within the Hepeviridae, the closest homologue to the HEV GI was the rat Orthohepevirus $\mathrm{C}$ macro domain $\left(\mathrm{E}\right.$-value $=1.73 \times 10^{-45} ;$ with $56 \%$ identity over 143 amino acids) (Table 1). The closest non-Hepeviridae homology was also seen between HEV and alphaviruses, such as Aura virus and Eastern equine encephalitis virus $\left(\right.$ EEEV) $\left(\mathrm{AF} 126284\right.$; $\mathrm{E}$-value $=4.61 \times 10^{-8}$, with $36 \%$ identity over 111 amino acids) (Table 1). Interestingly, the HEV macro domain also demonstrated similar identity with bacterial macro domains, such as that from Clostridium spiroforme $\left(\mathrm{E}\right.$-value $=1.62 \times 10^{-7}$, with $32 \%$ identity over 116 amino acids). The nearest eukaryotic homologue was the macro domain from the salmon flagellate protist pathogen Spironucleus salmonicida $($ EST47993; E-value $=$ $5.0 \times 10^{-6}$, with $32 \%$ identity over 108 amino acids).

\section{Helicase}

The HEV GI helicase protein (accession number NP056785) is also highly conserved within the Hepeviridae and has homology to non-Hepeviridae and non-viral helicases as well. The strongest non-human Hepeviridae Blast hit was found to be the helicase region of Orthohepevirus $\mathrm{C}$ ferret HEV ORF1 polyprotein (E-value = $7.0 \times 10^{-87}$, with $62 \%$ identity over 242 amino acids) (Table 1). The non-Hepeviridae viral helicase with the most identity to the HEV helicase was found to be that from the turnip vein clearing virus (Fig. 5), a Tobamovirus of the Virgaviridae family (E-value $=6.04 \times 10^{-13}$, with $32 \%$ identity over 250 amino acids). Another close homology was again observed in the helicase region from HASV (EU345431; E-value $=2.43 \times 10^{-12}$, with $32 \%$ identity over 250 amino acids) (Fig. 5).

\section{RNA-dependent RNA polymerase}

The HEV GI RNA-dependent RNA polymerase (RdRp; accession number NP056786) is highly conserved within the Hepeviridae, with $84-89 \%$ amino acid identity between the human HEV viruses, and 87-50 \% within nonhuman Hepeviridae viruses. The strongest identity was found with the RdRp region of Orthohepevirus $C$ ferret HEV polyprotein (JN998607; E-value $=3.64 \times 10^{-177}$, with $59 \%$ identity over 471 amino acids) (Table 1 ). The weakest Hepeviridae homology was with bat Orthohepevirus D and CTV, with $47 \%$ and $34 \%$ amino acid identity, respectively. The HEV RdRp also demonstrated homology to viral RdRps outside of the Hepeviridae family. Of the non-Hepeviridae homologues, the RdRp regions of two unclassified viruses had the highest identity to the human HEV RdRp; tick-borne tetravirus-like virus (KM048322; E-value $=7.82 \times 10^{-20}$, with $33 \%$ identity over 224 amino acids), and the algal virus Chara australis virus (JF824737; $2.98 \times 10^{-11}$, with $34 \%$ over 335 amino acids) $[25,26]$ (Fig. 6, Table 1). Other viral families also had RdRps that were highly homologous to HEV RdRp, including Betaflexiviridae and Alphatetraviridae (Fig. 6).

Another unclassified virus, Hemorrhagic kidney syndrome virus (HKSV) [32], (accession number AF030878), actually had higher homology to HEV RdRp (Fig. 6), than the above-mentioned Tick-borne tetravirus-like virus and Chara australis virus (E-value $=1.66 \times 10^{-23}$, with $39 \%$ identity over 155 amino acids). HKSV was first identified in salmon in 2000, and originally described as a "togavirus-like" virus [32]. This partial sequence has a recognizable $\mathrm{RdRp}$ domain with the characteristic 
RdRp nucleotide motifs B and catalytic motif C [33], albeit separated by a stop codon (Fig. 6).

\section{HEV ORF2 (Capsid) protein}

The Orthohepevirus A HEV GI capsid protein (encoded by ORF2; accession number NP056788) is well conserved within the Hepeviridae family, with the highest scoring non-human Hepeviridae homologue being the Orthohepevirus $\mathrm{C}$ ferret HEV capsid (AB890001; E-value =0, with $57 \%$ identity over 608 amino acids) (Fig. 7, Table 1). The next closest Orthohepevirus A homologue was that of bat Orthohepevirus D, (JQ001749; E-value $=3.94 \times 10^{-166}$, with $55 \%$ identity over 526 amino acids), followed by avian Orthohepevirus B (E-value $=6.55 \times 10^{-144}$, with $49 \%$ identity over 536 amino acids) (Fig. 7).

Interestingly, outside of the Hepeviridae, the chicken astrovirus capsid (JN582319; Avastrovirus, family Astroviridae) was found to be the most homologous sequence to the HEV GI capsid (E-value $=1.0 \times 10^{-6}$, with $25 \%$ identity over 278 amino acids) using a tBlastn search (Blosum62 matrix, word size 2, 3, or 6). Switching to the Blosum 45 matrix, for aligning more divergent sequences, resulted in porcine astrovirus capsid being the most homologous sequence identified (JX556690; E-value $=2.02 \times 10^{-12}$, with $21 \%$ identity over 355 amino acids) (Fig. 7, Table 1). Other astrovirus capsid sequences were also found to be similarly homologous (Fig. 7), with E-values ranging from $10^{-5}$ to $10^{-12}$, and percentage amino acid identity ranging from 20 $26 \%$ (data not shown). In addition, the unclassified virus discussed above, HKSV, had a similar level of homology with the HEV capsid to those of the astroviruses (E-value = $9.95 \times 10^{-5}, 26 \%$ identity over 164 amino acids). Additional PSI-Blast and Delta-Blast searches did not reveal any more significant homology to other capsid sequences from any other viral family or genera. A reciprocal tBlastn and PSIBlast search using the translated chicken astrovirus capsid sequence JN582319, identified HEV capsid protein as its closest homologue (27 \% identity over 278 amino acids; Evalue $=3.0 \times 10^{-6}$ ).

\section{HEV ORF3 phosphoprotein}

The function of the small phosphoprotein encoded by HEV ORF3 (accession number NP056877) is not fully understood, but it plays a role in virion release from infected cells, and has also recently been found to inhibit nuclear factor- $\mathrm{kB}$ signaling [34]. It is conserved within the human-infecting Orthohepevirus A genotypes, but not within the Hepeviridae family, even though viruses from the Orthohepevirus B, C and D, and CTV all have small, unrelated ORFs (presumed phosphoproteins) in the same part of their respective genomes. Delta-Blast and PSI-Blast searches did not reveal any other homologous proteins (viral or non-viral).

\section{Hepeviridae evolution - capsid and RdRp phylogeny indicates ancient recombination events}

An obvious disparity was revealed by the above analyses; the Orthohepevirus A HEV GI RdRp encoded from ORF1 is clearly related to viruses from families within the "alpha-like" supergroup, whilst the capsid encoded by ORF2 appears to be related to viruses of the Astroviridae family that falls within the "Picorna-like" supergroup of viruses. In order to understand these disparate homologies, a phylogenetic analysis of the HEV RdRp and capsid protein sequences was undertaken in relation to other viruses. RdRp and capsid sequences (Additional file 2: Table S2) from most of the type strains of class IV (+) ssRNA viruses were aligned by MAFFT, and phylogenetic relationships were inferred using PhyML to generate rooted and unrooted trees (Figs. 8 and 9).

The RdRp tree places the HEV RdRp and CTV RdRp together, forming a small cluster of RdRps with those from the Alphatetraviridae and Benyviridae families. The unclassified tick-borne tetravirus-like virus and Chara australis virus also groups with this cluster (Fig. 8). This small cluster's next nearest neighbor is RuBV. All of these group together, forming one of the main clusters within the "alpha-like" supergroup of viruses, which can be clearly seen in the unrooted tree (Fig. 9).

In contrast to the RdRp phylogeny, the rooted capsid tree places the HEV capsid within a cluster with the Astroviridae family of viruses (Fig. 8). The Astroviridae capsid is also closely related to the Tymoviridae family of monopartite, $\mathrm{T}=3$ icosahedral plant viruses. Although the HEV RdRp sequence groups with members of the Benyviridae and RuBV, there is no relationship between the Hepeviridae capsids and capsid sequences from these viruses, which group well away from the Astroviridae cluster (Fig. 8). Indeed, capsids from viruses of the Benyviridae are helical in architecture, not icosahedral. This relationship can also be seen more clearly in the unrooted capsid tree (Fig. 9). In order to reduce the potential effects of saturation [35] due to the large scale phylogeny of all $(+)$ ssRNA capsids (and their rapid mutation rates), an extended set of capsid sequences was analyzed again, this time including more Hepeviridae and Astroviridae sequences, but with fewer, more closely related families (Additional file 3: Figure S2). This analysis included 223 representatives from the Astroviridae, Benyviridae, Bromoviridae, Caliciviridae, Hepeviridae, Tombusviridae and Tymoviridae families (Additional file 2: Table S3). All of the families form individual clusters, except for the Bromoviridae strains. There is good branch support for several of the intrafamily clusters (Hepeviridae, Tymoviridae, Benyviridae and Caliciviridae), but this is weaker $(<60 \%)$ for the other intra- and interfamily clusters (Additional file 3: Figure S2). However, the Astroviridae and Hepeviridae group together, with the Tymoviridae 


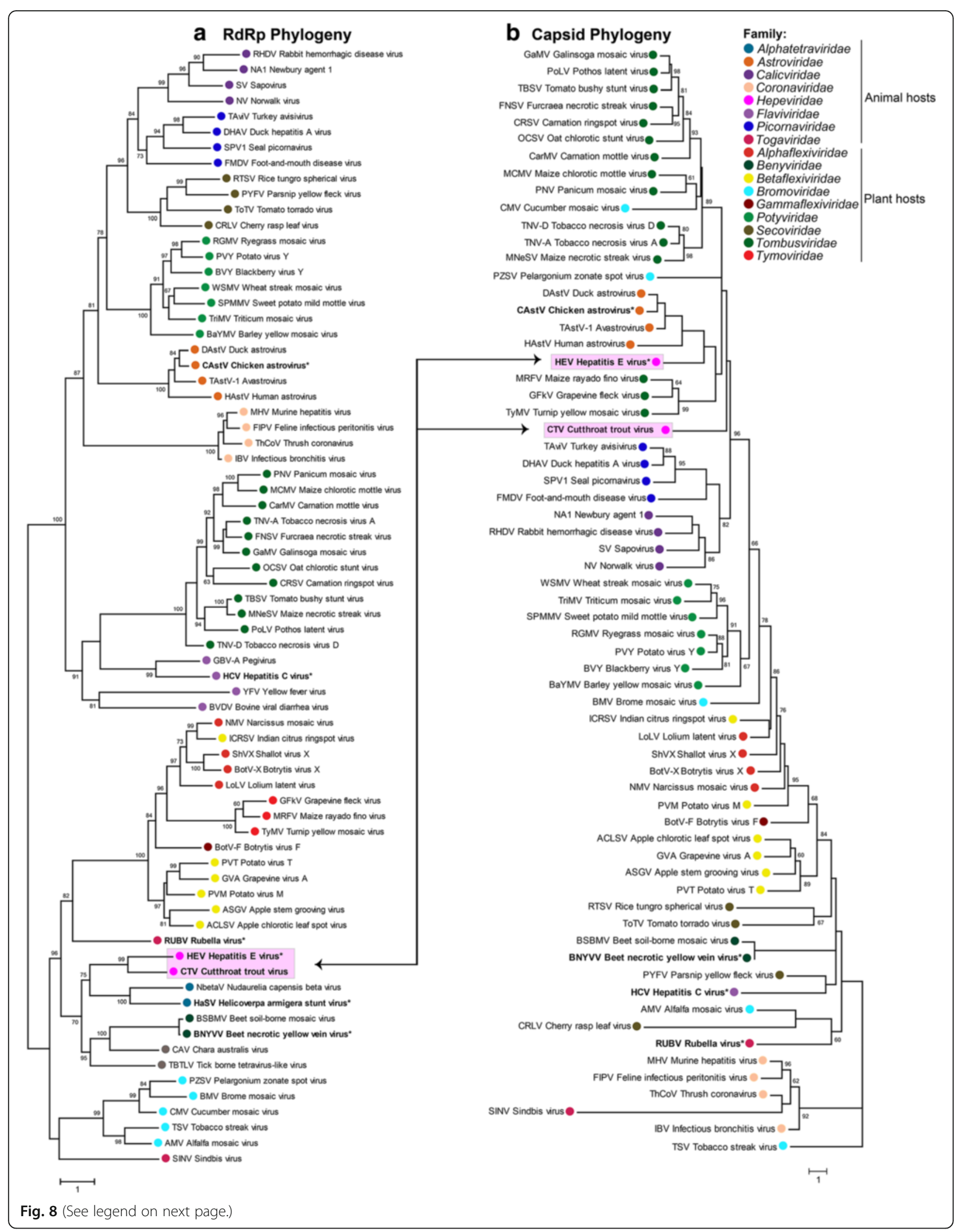


(See figure on previous page.)

Fig. 8 Rooted ssRNA (+) viruses capsid and RdRp phylogeny. a Midpoint rooted phylogenetic tree of 72 ssRNA (+) RdRp sequences, aligned by MAFFT and phylogeny performed using PhyML. Branch labels are bootstrap values. b Midpoint rooted phylogenetic tree of 65 ssRNA (+) capsid sequences, aligned by MAFFT and phylogeny performed using PhyML. Branch labels are bootstrap values. Recombination events within the Hepeviridae are shown by black arrows. Scale bars indicate number of nucleotide substitutions per site

linked to the Hepeviridae after apparent divergence from the Astroviridae cluster (Additional file 3: Figure S2).

\section{Discussion}

\section{Homology searches using the HEV GI proteins}

The first part of this study has highlighted four key areas that lead to a greater understanding of the origins and evolution of the Hepeviridae as a whole, with a focus on the prototype virus HEV GI in particular, and these are discussed in more detail below.

\section{MTase, Y-domain, helicase and RdRp}

The well-characterized domains with recognizable homology to other viral proteins include the MTase, Y-domain, helicase and RdRp domains. Of these, the Y-domain was originally identified as a distinct domain with weak homology to genomic regions in RuBV and BNYVV [8]. However, no function was ever ascribed to it. Since the preceding methyltransferase domain (NP056780) shares the same Pfam domain classification as the Ydomain, it seems more than likely that the Y-domain is actually just a C-terminal domain of a larger methyltransferase protein. Therefore, the MTase (NP056780) and Y- domain (NP056781) sequences should be considered together as one methyltransferase protein.

Recently, a secondary structure comparison of 50 viral genera identified an "iceberg" region, downstream of the "core" region within viral methyltransferaseguanylyltransferase (MTase-GTase) capping enzymes, to which the HEV Y-domain was also included [36]. Experimental evidence for the iceberg region or Y-domain's functional role has been demonstrated using C-terminal deletions of the Sindbis virus MTase-GTase [37]. Here, even relatively small C-terminal deletions abolished MTase-GTase activity, demonstrating that the core region alone is insufficient for all capping enzyme activities. This is further evidence that the MTase domain, together with the $\mathrm{Y}$ domain, forms either a single protein or single domain.

The individual searches with the Orthohepevirus A HEV ORF1 polyprotein and the individual MTase, Y-domain, helicase and RdRp proteins suggests that the majority of the ORF1 polyprotein of viruses from the Hepeviridae (comprising the MTase-Y-domain, helicase and RdRp domains) share a common ancestor with the Alphatetraviridae family, and that ancestor in turn shares a common



Fig. 9 Unrooted ssRNA (+) viruses Capsid and RdRp phylogeny. Unrooted phylogenetic tree of ssRNA (+) (a) RdRps and (b) capsids, aligned by MAFFT and phylogeny performed using PhyML. Shading indicates the viral supergroup to which clusters belong, as indicated by the key. Red arrows demonstrate the disparate genetic relationships for non-structural and structural proteins between supergroups, for viruses belonging Hepeviridae and Astroviridae. The capsid type of each cluster or group is depicted by an icon as indicated in the legend. Scale bars indicate number of nucleotide substitutions per site 
ancestor to the Virgaviridae/Benyviridae/Betaflexiviridae plant viral families. Other features of the HEV genome were probably acquired by horizontal gene transfer (polyproline-macro domain) or evolved later as a result of host-virus interactions (polyproline region).

\section{Papain-like cysteine protease, polyproline region, $\mathbf{Z}$ region and macro domain}

The putative papain-like cysteine protease domain, polyproline region, $\mathrm{Z}$ region and macro domain are the poorly characterized domains with little or no homology to other viral proteins. In the HEV genome these are grouped together, bisecting the four recognized proteins (Figs. 2 and 10). In addition, we recognized an uncharacterized region of 119 amino acids upstream of the polyproline region, which we have termed the $\mathrm{Z}$ region (Figs. 2 and 10), as there are already domains termed Macro (X) and Y present within the HEV genome.

The existence of the HEV protease was first predicted by Koonin et al. [8], based upon alignments with RuBV and BNYVV viruses. However, the alignment is poor, and subsequent advances in sequence alignment and enlarged databases have not led to the identification of any significant homologues to the HEV protease domain. Indeed, one of the most contentious issues regarding HEV is whether the ORF1 polyprotein is processed at all, either by its own protease or by a host protease. Evidence for in vitro processing of the HEV ORF1 has been reported in E. coli, in human HepG2 and S10-3 cells [38-40], in baculovirus cells [41] and using vaccinia virus expression systems [42]. However, in all of these studies, the levels of the putative processed proteins are low, and in some cases could be explained by host-cell protease degradation. A recombinant HEV protease purified from $E$. coli was found to be active on both the
HEV capsid protein and the HEV polyprotein, although an inhibitor screen of this protease's activity led to the conclusion that it was a chymotrypsin-like protease, not a papain-like cysteine protease [43]. However, other studies looking at ORF1 processing in human and hepatic cells reported little or no processing [44, 45]. This data is consistent with the fact that, a specific histidine residue (H590), a key part of the catalytic dyad in papain-like cysteine proteases, is absent in the HEV enzyme [46]. Pertilla et al. [45] also suggests that the HEV polyprotein is not processed, and functions in a similar manner to the large multifunctional polyproteins of some plant viruses within the "alpha-like" supergroup, consistent with its phylogenetic clustering (Figs. 8 and 9) [47]. Conversely, Parvez and colleagues used in silico modelling of RuBV and HEV proteases to identify a different catalytic dyad in the putative HEV papain-like protease. This study proposed that the catalytic dyad is C434-H443, which is homologous to the C1152-H1273 catalytic dyad in the RuBV protease [48]. Using mutational analyses, the conserved H443 was shown to be is essential for HEV viability [48]. In the current study, the H443 residue was found to be conserved within the Orthohepevirus A, but not across the Hepeviridae family. Irrespective of the functionality (or not) of the protease, its origin remains obscure due to the lack of any significant identity to non-Hepeviridae sequences.

From this study, it is also interesting to note that this "protease" region, which lies between the MTase-GTase and the polyproline region, is approximately the same size across members of the Hepeviridae. Yet the amino acid identity is so low that no genetic relationship is apparent (Additional file 1: Figure S1). This suggests a common functional enzyme does not exist. Instead, perhaps this is a region involved in host-specific interactions.




Whether this domain is a degenerate partially functional or non-functional protease domain, or an entirely different domain or protein, remains to be clarified.

One other study has reported deubiquitination activity, a key target for viral infection [49], associated with a recombinant protein comprising the methyltransferase, Y-domain and protease domain (amino acids 22-592) of HEV ORF1 [50]. A later study showed that a recombinant HEV protease domain, when expressed in HEK293T cells, resulted in reduced IFN $\beta$ mRNA production and deubiquitination of RIG-I and TBK-1 [51]. Since cysteine proteases are significantly represented as a subfamily within the larger deubiquitinase (DUB) enzyme family [52], this could well be the main function, even perhaps the only function, for the protease domain.

The macro domain of HEV is homologous to those found in alphaviruses and bacteria, with slightly less similarity to eukaryotic and archeal macro domains, but the differences in homology are not significant enough to identify unambiguously the origin or common ancestor of this region. It is also unclear whether the HEV macro and protease domains together derive from the same ancestral region, as is the case for the Coronaviridae and alphaviruses [14], where the macro domain is associated with the protease domain. Macro domains in animal cells have been associated with ADP-ribose metabolism, and post translational protein modification, but few viral families possess macro domains, and in those viral families that do, the macro domains do not appear to be related to any specific feature shared by these viruses (Coronaviridae, alphaviruses, Hepeviridae) [31]. Poly(ADP)-ribosylation is a post-translational modification of proteins in cells, which can be triggered by viral infection, and acts as an apoptosis-inducing signal [53], and the HEV macro domain has been shown to bind poly(ADP), suggesting a potential role in preventing apoptosis in infected cells. Expression of a recombinant HEV macro domain in HEK293T cells inhibited poly(IC)-induced IRF3 phosphorylation, indicating that one of the HEV macro domain's function is as an IFN antagonist [51].

The polyproline region (PPR) was originally described as a protein hinge [8], but more recent studies have shown it to be the only region within ORF1 under positive selection, and has multiple ligand-binding motifs [29]. The PPR is also involved in viral replication and adaptation, including host-specificity, but not infectivity $[54,55]$. An increase in correlated genetic heterogeneity in the macro and polyproline regions has been observed in chronic HEV infection, relative to patients who cleared the virus, suggesting that these two domains are linked in some way in modulating the immune response [56]. Apart from the Hepeviridae, only rubiviruses have a similar arrangement of a PPR upstream of a macro domain, and the rubivirus PPR is also highly diverse genetically, and under positive selection [29]. It is conceivable that ancestral rubiviruses and Hepeviridae viruses at some point acquired or exchanged a PPR-macro domain, which has retained homology in the macro domain, for its shared function in host-virus interactions, but the PPR domain has evolved rapidly due to its host-specificity role.

The $\mathrm{Z}$ region of the Orthohepevirus A HEV GI ORF1 is unique across the species and as such, resembles an equivalent alphavirus unique domain (AUD); a region of the alphaviral non-structural protein coding region that has strong sequence identity within alphaviruses, but not outside the genus [57]. Like the AUD, the $\mathrm{Z}$ region is highly variable in both sequence and length outside Orthohepevirus A, but is conserved within the Orthohepevirus A group ( $>68 \%$ identity across 119 residues). It is possible that the AUD-like region in Orthohepevirus A viruses has evolved with changes in host tropism, thus losing any conservation with other members of the Hepeviridae over time. Similar to the $\mathrm{Z}$ region in Orthohepevirus $\mathrm{A}$, in alphaviruses a PPR follows the AUD, however unlike HEV both domains are downstream of the macro domain (Fig. 2). In the majority of Orthohepevirus $\mathrm{A}$ sequences, the $\mathrm{Z}$ region and PPR are both upstream of the macro domain and could have shifted through a translocation event. One exception within Orthohepevirus A is rabbit HEV, and outside Orthohepevirus A exceptions include ferret and rat HEV (Orthohepevirus $\mathrm{C}$ ) and CTV (Piscihepevirus) which all have a small PPR region at the carboxyl end of the macro domain. This polyproline rich region was originally reported to be an insertion of an inert spacer sequence into the rabbit and rat genomes [58], but could in fact be a remnant from an incomplete PPR translocation event.

\section{Capsid}

Whereas the majority of the HEV ORF1-encoded polyprotein and those from the Hepeviridae family in general clearly have plant/insect viral ancestry, the HEV capsid sequence appears to have none. Analysis of the capsid spike or projection domain of the human astrovirus serotype 8 (human astrovirus; Yuc8 strain, genus Mamastrovirus) using the DALI protein homology server [59] identified the HEV capsid P2 domain as the closest structural homologue [23].

Electron cryomicroscopy also showed that the human astrovirus infectious virions and immature virions were remarkably similar to that of $\mathrm{HEV}$ in terms of size, shape and architecture [22,60]. It is interesting to note that, given the similarity in capsid structural size, the genomes of HEV $(\sim 7.2 \mathrm{~kb})$ and astroviruses $(6.8-7.1 \mathrm{~kb})$ are also similarly matched in size. A subsequent study [61] found that the turkey astrovirus spike protein (turkey astrovirus-2; genus Avastrovirus) was a structurally closer match to the HEV capsid, than was found 
with the previous study involving the human astrovirus capsid spike [60]. The present study however, showed that the porcine astrovirus capsid protein sequence was the closest homologue to the HEV capsid sequence, closely followed by the chicken astrovirus capsid (Fig. 7), and all the homologous Astroviridae capsids sequences had $\mathrm{E}$ values between $10^{-5}-10^{-12}$. Moreover, no other viral family capsid sequences had any significant homology to the HEV capsid. A reciprocal Blast search using a chicken astrovirus capsid sequence identified the HEV capsid as the closest homologue, and no other viral family capsid had any significant identity. Irrespective of which contemporary astrovirus sequence is most closely related to contemporary HEV capsids, it seems that the Hepeviridae and the Astroviridae viruses share a common ancestral capsid sequence.

Surprisingly, it was also found that the Tymoviridae and Hepeviridae capsid sequences have a common ancestor too, which diverged after the Hepeviridae-Astroviridae split (Additional file 3: Figure S2). However, the Hepeviridae and Tymoviridae capsid sequences do not have any significant homology, but are linked phylogenetically, which could be indicative of the (plant-infecting) Tymoviridae capsid sequences evolving faster under different evolutionary pressure(s), than the avian/mammalian-infecting Hepeviridae or Astroviridae capsids.

\section{ORF3 Phosphoprotein}

The absence of any viral homologues to the phosphoprotein encoded by ORF3, even within the Hepeviridae would suggest that ORF3 evolved relatively recently from an overprinting mechanism [62], utilizing a previously unused ORF within ORF2. ORFs 2 and 3 are bicistronic, with the two start codons closely spaced in different reading frames [63]. ORF3 has evolved to be essential for virulence [64]. Viruses with this type of ORF3 likely occurred after mammalian Orthohepevirus A virus diverged from the common Hepeviridae ancestor and it is possible that ORF3 could have driven host tropism and host restriction [65]. Similar overprinted genes like ORF3, have been identified in numerous other viruses [66]. However, Astroviridae capsid sequences have not been shown to have overlapping proteins expressed from alternative ORFs, and it is seems highly improbable that ORF3 was independently acquired and integrated into the capsid sequence.

\section{Proposed evolutionary scenario for the Hepeviridae}

There are few reports in the literature regarding the evolution of contemporary viruses from two or more disparate ancestors. Although there are few examples, one in particular is remarkable; the ssDNA viruses of the Bidnaviridae family (infecting the silkworm Bombyx mori) contain genes derived from Parvoviridae (ssDNA viruses), Reoviridae (dsRNA viruses), Baculoviridae (dsDNA viruses) and polintoviruses [67]. In addition, a recent metagenomics study of viral diversity in a lake has identified a unique hybrid virus, where an ssRNA viral capsid sequence has integrated into a circovirus-like DNA virus (RNA-DNA hybrid virus from Boiling Springs Lake, or "BSL-RDHV") [68]. The capsid sequence in BSL-RDHV is most closely related to the capsids from multipartite nodaviruses, which mostly infect insects and fish, and to monopartite Tombusviridae capsids (plant viruses), and the remainder of the BSL-RDHV genome resembles circoviruses (infecting birds and mammals) [68]. RNA-RNA recombination in plant viruses and other viruses, and the relevant molecular mechanisms are well-known and well-documented [69]. Some families show high rates of recombination (Picornaviridae), and others low rates (Flaviviridae), or non-existent/undetectable (Leviviridae), nonetheless recombination is vital for generating population variability and maximizing survival [70].

There are few if any published reports of interfamily capsid exchange in the ssRNA (+) viruses, notwithstanding the fact that such an exchange would in all likelihood require similar sized genomes in order to package properly. The most parsimonious evolutionary scenario, with regard to the exchange, or acquisition of capsid sequences by an ancestral Hepeviridae virus, might be that an ancient ORF1 RNA sequence, from a plant/insect virus ancestral to the Alphatetraviridae/Betaflexiviridae/ Virgaviridae/Benyviridae families, underwent recombination with an ancestral Astroviridae capsid RNA sequence, resulting in a recombinant virus that had (fortuitously) similarly sized genome of a plant/insect virus. This virus likely retained capsid sequence packing signals and was able to be subsequently packaged into an astrovirus-like icosahedral capsid. Such a recombination event resulted in the ancestral Hepeviridae hybrid virus, comprising an astroviral capsid from the Picornalike super-group, and a plant/insect non-structural polyprotein from a virus within the very different alpha-like super-group (Fig. 10). An obvious prerequisite of the above events is that the ancestral viruses involved infected the same host simultaneously; whether this permissive host was a plant, insect or possibly even a vertebrate is at present unclear.

The ancestral Hepeviridae ORF1 probably comprised (from $\mathrm{N}$ - to C-terminal) a methyltransferase, "protease", helicase and RdRp regions. At some time a macro domain was acquired, probably by horizontal transfer, although the putative source is unclear, given the similar, (albeit low) identity to bacterial and alphaviral homologues. Whether this acquisition occurred before, or after the capsid exchange is unclear. This acquisition may have been accompanied by the "protease" region, since these two domains are often closely associated, although no functional link between these domains has 
yet been demonstrated [14]. The PPR could also have been associated with the macro domain as discussed above. The orientation of the protease and macro domains in Hepeviridae viruses correspond to that seen in alphaviruses, but is the reverse of RuBV, whereas the PPRmacro domain organization in Hepeviridae matches that of rubiviruses, but not alphaviruses in general (which have a much smaller PPR region or lack the PPR altogether) $[8,47]$ (Fig. 10). Later still, the ORF3 phosphoprotein evolved from an alternate reading frame within the capsid sequence, which could have been present in the capsid region prior to recombination, or evolved afterwards. Subsequently, the evolution of ORF3 could have been influenced by host tropism, leading to the possible evolution of individual species within the Hepeviridae.

Intriguingly, duck astrovirus is unique among the Astroviridae, in causing viral hepatitis, indicating a tropism for hepatocytes, whereas all other astroviruses cause gastroenteritis [71]. It is interesting to speculate that the ancestral virus, from which viruses of the Astroviridae and Hepeviridae obtained their capsids, had a similar tropism. This could help to explain how the descendent capsid of an enteric virus is now used by a virus that causes human hepatitis.

It is also noteworthy that analysis of the Astroviridae capsid and RdRp sequences indicate the possible occurrence of a second ancient inter-viral family recombination event. The astrovirus RdRp sequences are unrelated to those from the Hepeviridae family, but are most closely related to those from viruses of the Potyviridae; a large family of non-enveloped plant-infecting viruses [72] with a flexible filamentous rod-shaped virion (Figs. 8 and 9). These capsids share no homology whatsoever with the icosahedral astrovirus capsid. This likely indicates that an ancestor of the Potyviridae acquired a distinct Astroviridae-like capsid (i.e., from a different viral family), indicating a recombination event at the non-structural/structural junction, that led to the genesis of the Astroviridae.

Koonin et al., [8] first proposed that HEV (and hence subsequently the whole family) had a genetic relationship in the non-structural encoding region of its genome with viruses from the Togaviridae, including $\mathrm{RuBV}$ and BNYYV; this is depicted in Fig. 10. In contrast to the Hepeviridae, the Togaviridae (including RuBV), have an alpha-like non-structural genomic region, but a flavivirallike structural region of their genome (Fig. 10). Given that the two aforementioned recombination predictions likely led to the genesis of the Hepeviridae and the Astroviridae, it is tempting to speculate that the entire Togaviridae family could also have emerged this way. It may have occurred through a recombination event at the non-structural/ structural border between viruses from families representing different viral super-groups; flavi-like and alpha-like super-groups.

\section{Conclusion}

This study used protein identity searches using the prototype HEV virus ORF1 amino acid sequence to highlight four key areas that lead to a greater understanding of the origins of HEV, the possible functions of its encoded proteins and their relationship to other protein families. This study has also shed additional light on the intriguing evolution and ancestry of several viral families. We show that both the Astroviridae and Hepeviridae, and possibly the Togaviridae, have undergone ancient recombination events at the junction between the non-structural and structural encoding regions of the genome, resulting in their creation. It is likely other families have emerged this way and this could be uncovered with careful analysis of the ever-growing number of new viral sequences being accrued. Finally, based on the results found herein, we hypothesize that viruses from the alpha-like super-group, which usually infect plants and insects, gain an entirely new structure through recombination to enable tropism into new hosts within the animal world.

\section{Methods \\ Hepeviridae genomic sequences}

A total of 301 Hepeviridae genomes were identified and downloaded from GenBank (October, 2015). Duplicate sequences were removed using the ElimDupes tool on the HIV sequence database (https://hcv.lanl.gov/content/sequence/ELIMDUPES/elimdupes.html). Orthohepevirus B, $\mathrm{C}$ and D genomes, along with the Piscihepevirus CTV genome, were removed. Rabbit HEV (GIII), wild boar HEV (GV), and camel HEV (GVII) sequences were included, but wild boar HEV (GVI) and Moose HEV (unclassified) sequences were omitted. Incomplete sequences, patent sequences of cDNA, recombinant infectious clone constructs and sequences from ex vivo passaged isolates were also excluded, resulting in 228 non-redundant HEV genomes (Additional file 2: Table S1).

\section{Identity searching of Orthohepevirus A HEV GI proteins}

To identify homologous Hepeviridae and non-Hepeviridae viral sequences, individual Orthohepevirus A HEV GI protein sequences were used in tBlastn and Blastp searches of the GenBank databases [73], using the built-in interface in the software package Geneious 9.0.3 [74]. Specifically, protein sequences derived from the prototype HEV Xinjiang strain (accession number NC_001434) [75] were used, corresponding to the methyltransferase (NP056780), Y-domain (NP056781), papain-like cysteine protease (NP056782), hypervariable region (HVR) or polyproline region (NP056783), X (macro) domain (NP056784), helicase (NP056785) and RNA-dependent RNA polymerase proteins (RdRp, NP056786), shown together in Fig. 2. In addition, searches were also 
conducted with the complete polyprotein of ORF1 (NP056779), the capsid protein from ORF2 (NP056788) and the phosphoprotein encoded by ORF3 (NP056787) [75]. An "expectation" or "expect value" (E-value) threshold of $<1 \times 10^{-4}$ was used to eliminate weak and random similarities between the query and target sequences [76].

\section{Homology of HEV GI protein sequences searching by PSI-Blast, Delta-Blast and InterProScan}

The individual human HEV GI protein sequences and the HEV ORF1 polyprotein sequences described above (Fig. 2), were also used to screen the non-redundant GenBank protein database for distant homologues, using the position-specific iterated Blast (PSI-BLAST) server at http://blast.ncbi.nlm.nih.gov/ [77]. Initial setup parameters comprised the default settings, using either BLOSUM45 or BLOSUM62 as the initial substitution matrix, word size 2 or 3 , up to 5 iterations, and masking of low complexity regions. In addition, searches were made using the same parameters on the Domain Enhanced Lookup Time Accelerated Blast, or Delta-Blast server. This server queries the NCBI conserved domain database (CDD) to construct a scoring matrix prior to searching the protein sequence database [78]. Individual HEV GI protein sequences were also analyzed for protein function against the InterPro protein families' database, using the InterProScan plugin in Geneious [79, 80].

\section{Multiple sequence alignment and phylogenetic analysis} Capsid and RdRp protein sequences from 104 representative positive sense, single stranded RNA viruses (ssRNA (+)) were collated from GenBank (Additional file 2: Table S2). These sequences were aligned using MAFFT [81], using the embedded algorithms in Geneious. The best-fit models for amino acid substitution for the phylogenetic analysis were determined by analyzing the capsid and RdRp alignments using ProtTest 3.3 [82]. Phylip files of the alignments were submitted to the ATGC Server at http://www.atgc-montpellier.fr/ to build the phylogeny using PhyML [83], using the LG substitution model [84] for RdRp alignments, and the VT substitution model for capsid alignments [85], with SPR and NNI tree improvements and 100 bootstraps.

\section{Additional files}

Additional file 1: Figure S1. Hepeviridae protease alignment. Alignment of the $3^{\prime}$ terminal of the $Y$-domain, protease domain and polyproline region of HEV GI and the three closest Hepeviridae homologues, using MAFFT. (TIF $2501 \mathrm{~kb}$ )

Additional file 2: Table S1. List of Hepeviridae genomes analyzed. Table S2. List of capsid and RdRp sequences used for Figs. 8 and 9. Table S3. List of 223 genomic sequences from capsid sequences used for creating additional Fig. 2. (DOCX $122 \mathrm{~kb}$ )
Additional file 3: Figure S2. Extended capsid phylogeny. Midpoint rooted maximum likelihood phylogenetic tree of 223 capsid sequences (Additional file 2: Table S3) from seven families, aligned by MAFFT and phylogeny performed using PhyML. Red values: bootstrap scores > $60 \%$; Black values: substitutions per site. (TIF $914 \mathrm{~kb}$ )

\section{Acknowledgements}

The authors wish to thank Associate Professor Mark Tanaka, Dr. John-Sebastian Eden and Professor Eddie Holmes for very useful discussions.

\section{Funding}

No specific funding was received for this study. NEN was supported by an Australian Postgraduate Award.

\section{Availability of data and material}

The datasets analysed during the current study are available from the corresponding author on reasonable request.

\section{Authors' contributions}

AGK, NEN \& PAW conceived the study; AGK \& PAW designed the study; AGK, NEN \& PAW carried out the work, data analysis and drafted the manuscript; AGK, NEN \& PAW contributed to interpretation of data; AGK, NEN \& PAW critically revised the manuscript. All authors read and approved the final manuscript.

\section{Competing interests}

The authors declare that they have no competing interests.

\section{Consent for publication}

Not applicable.

\section{Ethics approval and consent to participate}

Not applicable.

Received: 29 March 2016 Accepted: 30 September 2016 Published online: 12 October 2016

\section{References}

1. Purcell RH, Emerson SU. Hepatitis E: an emerging awareness of an old disease. J Hepatol. 2008:48(3):494-503.

2. World Health Organization (WHO): Hepatitis E — fact sheet No 280 [http://www.who.int/mediacentre/factsheets/fs280/en/]. Accessed Aug 2016

3. Rein DB, Stevens GA, Theaker J, Wittenborn JS, Wiersma ST. The global burden of hepatitis E virus genotypes 1 and 2 in 2005. Hepatology. 2012;55(4):988-97.

4. Pischke $\mathrm{S}$, Wedemeyer $\mathrm{H}$. Hepatitis $\mathrm{E}$ virus infection: multiple faces of an underestimated problem. J Hepatol. 2013;58(5):1045-6.

5. Pischke $\mathrm{S}$, Behrendt $\mathrm{P}$, Bock $C T$, Jilg W, Manns MP, Wedemeyer $\mathrm{H}$. Hepatitis E in Germany-an under-reported infectious disease. Dtsch Arztebl Int. 2014; 111(35-36):577-83.

6. Smith DB, Simmonds P, Jameel S, Emerson SU, Harrison TJ, Meng XJ, Okamoto H, Van der Poel WH, Purdy MA. Consensus proposals for classification of the family Hepeviridae. J Gen Virol. 2014;95(Pt 10):2223-32.

7. Lin J, Norder $\mathrm{H}$, Uhlhorn $\mathrm{H}$, Belak S, Widen F. Novel hepatitis E like virus found in Swedish moose. J Gen Virol. 2014;95(Pt 3):557-70.

8. Koonin EV, Gorbalenya AE, Purdy MA, Rozanov MN, Reyes GR, Bradley DW. Computer-assisted assignment of functional domains in the nonstructural polyprotein of hepatitis E virus: delineation of an additional group of positive-strand RNA plant and animal viruses. Proc Natl Acad Sci U S A. 1992;89(17):8259-63.

9. Canova A. Si studia la rizomania della bietola. Informatore Fitopatalogica. 1966;16:235-9.

10. Tamada T, Baba T. Beet necrotic yellow vein virus from Rizomania-affected sugar beet in Japan. Jpn J Phytopathol. 1973;39(4):325-32. 321.

11. Abe H, Tamada T. Association of beet necrotic yellow vein virus with isolates of Polymyxa betae Keskin. Jpn J Phytopathol. 1986;52(2):235-47.

12. Tamada T, Shirako Y, Abe H, Saito M, Kiguchi T, Harada T. Production and Pathogenicity of Isolates of Beet Necrotic Yellow Vein Virus with Different Numbers of RNA Components. J Gen Virol. 1989;70(12):3399-409.

13. Koonin EV. The phylogeny of RNA-dependent RNA polymerases of positive-strand RNA viruses. J Gen Virol. 1991;72(9):2197-206. 
14. Gorbalenya AE, Koonin EV, Lai MM. Putative papain-related thiol proteases of positive-strand RNA viruses. Identification of rubi- and aphthovirus proteases and delineation of a novel conserved domain associated with proteases of rubi-, alpha- and coronaviruses. FEBS Lett. 1991;288(1-2):201-5.

15. Cubitt W BD, Carter M, Chiba S, Estes M, Saif L, Schaffer FL, Smith AW, Studdert MJ, Thiel HJ: Caliciviridae. In: "Virus Taxonomy: The sixth report of the international committee for taxonomy of viruses", vol. 10. Vienna: Springer-Verlag; 1995

16. Berke T, Golding B, Jiang X, Cubitt DW, Wolfaardt M, Smith AW, Matson DO. Phylogenetic analysis of the caliciviruses. J Med Virol. 1997;52(4):419-24.

17. Berke T, Matson DO. Reclassification of the Caliciviridae into distinct genera and exclusion of hepatitis $E$ virus from the family on the basis of comparative phylogenetic analysis. Arch Virol. 2000;145(7):1421-36.

18. Emerson SU, Anderson D, Arankalle A, Meng XJ, Purdy M, Schlauder GG, Tsarev SA. Hepevirus. Virus taxonomy. 2004:853-7.

19. King AM. Virus taxonomy: classification and nomenclature of viruses: Ninth Report of the International Committee on Taxonomy of Viruses. Volume 9. Elsevier; 2011.

20. Smith DB, Simmonds P, Izopet J, Oliveira-Filho EF, Ulrich RG, Johne R, Koenig M, Jameel S, Harrison TJ, Meng XJ, et al. Proposed reference sequences for hepatitis E virus subtypes. J Gen Virol. 2016;97(3):537-42.

21. Xing L, Kato K, Li T, Takeda N, Miyamura T, Hammar L, Cheng RH. Recombinant hepatitis E capsid protein self-assembles into a dual-domain $T=1$ particle presenting native virus epitopes. Virology. 1999;265(1):35-45.

22. Xing L, Li TC, Mayazaki N, Simon MN, Wall JS, Moore M, Wang CY, Takeda N, Wakita T, Miyamura T, et al. Structure of hepatitis E virion-sized particle reveals an RNA-dependent viral assembly pathway. J Biol Chem. 2010;285(43):33175-83.

23. Dong J, Dong L, Méndez E, Tao Y. Crystal structure of the human astrovirus capsid spike. Proc Natl Acad Sci U S A. 2011;108(31):12681-6.

24. Liu H, Fu Y, Jiang D, Li G, Xie J, Peng Y, Yi X, Ghabrial SA. A novel mycovirus that is related to the human pathogen Hepatitis $E$ virus and rubi-like viruses. J Virol. 2009;83(4):1981-91.

25. Tokarz R, Williams SH, Sameroff S, Sanchez Leon M, Jain K, Lipkin WI. Virome analysis of Amblyomma americanum, Dermacentor variabilis, and Ixodes scapularis ticks reveals novel highly divergent vertebrate and invertebrate viruses. J Virol. 2014;88(19):11480-92.

26. Gibbs AJ, Torronen M, Mackenzie AM, Wood JT, Armstrong JS, Kondo H, Tamada T, Keese PL. The enigmatic genome of Chara australis virus. J Gen Virol. 2011;92(11):2679-90.

27. Mistry J, Finn RD, Eddy SR, Bateman A, Punta M. Challenges in homology search: HMMER3 and convergent evolution of coiled-coil regions. Nucleic Acids Res. 2013:41(12):e121.

28. Vasilakis N, Forrester NL, Palacios G, Nasar F, Savji N, Rossi SL, Guzman H, Wood TG, Popov V, Gorchakov R, et al. Negevirus: a proposed new taxon of insectspecific viruses with wide geographic distribution. J Virol. 2013;87(5):2475-88.

29. Purdy MA, Lara J, Khudyakov YE. The hepatitis E virus polyproline region is involved in viral adaptation. PLoS One. 2012;7(4):e35974.

30. Purdy MA. Evolution of the hepatitis E virus polyproline region: order from disorder. J Virol. 2012;86(18):10186-93.

31. Egloff M-P, Malet $H$, Putics Á, Heinonen M, Dutartre $H$, Frangeul A, Gruez A, Campanacci V, Cambillau C, Ziebuhr J, et al. Structural and functional basis for ADP-Ribose and Poly(ADP-Ribose) binding by viral macro domains. J Virol. 2006;80(17):8493-502.

32. Kibenge FS, Whyte SK, Hammell KL, Rainnie D, Kibenge MT, Martin CK. A dual infection of infectious salmon anaemia (ISA) virus and a togavirus-like virus in ISA of Atlantic salmon Salmo salar in New Brunswick, Canada. Dis Aquat Organ. 2000;42(1):11-5.

33. Kamer G, Argos P. Primary structural comparison of RNA-dependent polymerases from plant, animal and bacterial viruses. Nucleic Acids Res. 1984;12(18):7269-82.

34. Xu J, Wu F, Tian D, Wang J, Zheng Z, Xia N. Open reading frame 3 of genotype 1 hepatitis $E$ virus inhibits nuclear factor-kappa B signaling induced by tumor necrosis factor-alpha in human A549 lung epithelial cells. PLoS One. 2014;9(6):e100787.

35. Moreira D, Philippe H. Molecular phylogeny: pitfalls and progress. Int Microbiol. 2000;3(1):9-16

36. Ahola T, Karlin DG. Sequence analysis reveals a conserved extension in the capping enzyme of the alphavirus supergroup, and a homologous domain in nodaviruses. Biology Direct. 2015;10:16.
37. Wang H-L, O'Rear J, Stollar V. Mutagenesis of the Sindbis Virus nsP1 Protein: Effects on Methyltransferase Activity and Viral Infectivity. Virology. 1996; 217(2):527-31

38. Ansari IH, Nanda SK, Durgapal H, Agrawal S, Mohanty SK, Gupta D, Jameel S, Panda SK. Cloning, sequencing, and expression of the hepatitis E virus (HEV) nonstructural open reading frame 1 (ORF1). J Med Virol. 2000;60(3):275-83.

39. Panda SK, Ansari IH, Durgapal H, Agrawal S, Jameel S. The in vitrosynthesized RNA from a cDNA clone of hepatitis E virus is infectious. J Virol. 2000;74(5):2430-7.

40. Parvez MK. Molecular characterization of hepatitis E virus ORF1 gene supports a papain-like cysteine protease (PCP)-domain activity. Virus Res. 2013;178(2):553-6.

41. Sehgal D, Thomas S, Chakraborty M, Jameel S. Expression and processing of the Hepatitis E virus ORF1 nonstructural polyprotein. Virol J. 2006;3:38.

42. Ropp SL, Tam AW, Beames B, Purdy M, Frey TK. Expression of the hepatitis E virus ORF1. Arch Virol. 2000;145(7):1321-37.

43. Paliwal D, Panda SK, Kapur N, Varma SPK, Durgapal H. Hepatitis E virus (HEV) protease: a chymotrypsin-like enzyme that processes both non-structural (pORF1) and capsid (pORF2) protein. J Gen Virol. 2014;95(Pt 8):1689-700.

44. Suppiah S, Zhou Y, Frey TK. Lack of processing of the expressed ORF1 gene product of hepatitis E virus. Virol J. 2011;8:245.

45. Perttila J, Spuul P, Ahola T. Early secretory pathway localization and lack of processing for hepatitis E virus replication protein pORF1. J Gen Virol. 2013; 94(Pt 4):807-16.

46. Ahmad I, Holla RP, Jameel S. Molecular virology of hepatitis E virus. Virus Res. 2011;161(1):47-58.

47. van der Heijden MW, Bol JF. Composition of alphavirus-like replication complexes: involvement of virus and host encoded proteins. Arch Virol. 2002;147(5):875-98.

48. Parvez MK, Khan AA. Molecular modeling and analysis of hepatitis $E$ virus (HEV) papain-like cysteine protease. Virus Res. 2014;179:220-4.

49. Lindner HA. Deubiquitination in virus infection. Virology. 2007:362(2):245-56

50. Karpe YA, Lole KS. Deubiquitination activity associated with hepatitis E virus putative papain-like cysteine protease. J Gen Virol. 2011:92(Pt 9):2088-92.

51. Nan Y, Yu Y, Ma Z, Khattar SK, Fredericksen B, Zhang YJ. Hepatitis E virus inhibits type I interferon induction by ORF1 products. J Virol. 2014;88(20):11924-32.

52. Amerik AY, Hochstrasser M. Mechanism and function of deubiquitinating enzymes. Biochimica et Biophysica Acta (BBA) - Mol Cell Res. 2004;1695(1-3): 189-207.

53. Hay S, Kannourakis G. A time to kill: viral manipulation of the cell death program. J Gen Virol. 2002;83(Pt 7):1547-64.

54. Pudupakam RS, Huang YW, Opriessnig T, Halbur PG, Pierson FW, Meng XJ. Deletions of the Hypervariable Region (HVR) in open reading frame 1 of hepatitis $E$ virus do not abolish virus infectivity: evidence for attenuation of HVR deletion mutants in vivo. J Virol. 2009:83(1):384-95.

55. Pudupakam RS, Kenney SP, Córdoba L, Huang Y-W, Dryman BA, LeRoith T, Pierson FW, Meng X-J. Mutational analysis of the hypervariable region of hepatitis $E$ virus reveals its involvement in the efficiency of viral RNA replication. J Virol. 2011:85(19):10031-40.

56. Lhomme S, Garrouste C, Kamar N, Saune K, Abravanel F, Mansuy J-M, Dubois M, Rostaing L, Izopet J. Influence of polyproline region and macro domain genetic heterogeneity on HEV persistence in immunocompromised patients. J Infect Dis. 2014:209(2):300-3.

57. Rupp JC, Sokoloski KJ, Gebhart NN, Hardy RW. Alphavirus RNA synthesis and non-structural protein functions. J Gen Virol. 2015:96(9):2483-500.

58. Smith DB, Vanek J, Ramalingam S, Johannessen I, Templeton K, Simmonds P. Evolution of the hepatitis E virus hypervariable region. J Gen Virol. 2012; 93(Pt 11):2408-18.

59. Holm L, Rosenström P. Dali server: conservation mapping in 3D. Nucleic Acids Res. 2010;38 suppl 2:W545-9.

60. Dryden KA, Tihova M, Nowotny N, Matsui SM, Mendez E, Yeager M. Immature and mature human astrovirus: structure, conformational changes, and similarities to hepatitis E virus. J Mol Biol. 2012;422(5):650-8.

61. DuBois RM, Freiden P, Marvin S, Reddivari M, Heath RJ, White SW, SchultzCherry S. Crystal structure of the avian astrovirus capsid spike. J Virol. 2013; 87(14):7853-63.

62. Grasse PP. Overlapping genes: a priority. C R Acad Sci Hebd Seances Acad Sci D. 1977:284(2):141-2.

63. Graff J, Torian U, Nguyen H, Emerson SU. A Bicistronic Subgenomic mRNA Encodes both the ORF2 and ORF3 Proteins of Hepatitis E Virus. J Virol. 2006; 80(12):5919-26. 
64. Yamada K, Takahashi M, Hoshino Y, Takahashi H, Ichiyama K, Nagashima S, Tanaka T, Okamoto H. ORF3 protein of hepatitis $\mathrm{E}$ virus is essential for virion release from infected cells. J Gen Virol. 2009;90(8):1880-91.

65. Bouquet J, Cherel P, Pavio N. Genetic characterization and codon usage bias of full-length Hepatitis $E$ virus sequences shed new lights on genotypic distribution, host restriction and genome evolution. Infect Genet Evol. 2012; 12(8):1842-53.

66. Sabath N, Wagner A, Karlin D. Evolution of viral proteins originated de novo by overprinting. Mol Biol Evol. 2012;29(12):3767-80.

67. Krupovic M, Koonin EV. Evolution of eukaryotic single-stranded DNA viruses of the Bidnaviridae family from genes of four other groups of widely different viruses. Sci Rep. 2014;4:5347.

68. Diemer GS, Stedman KM. A novel virus genome discovered in an extreme environment suggests recombination between unrelated groups of RNA and DNA viruses. Biol Direct. 2012;7:13.

69. Sztuba-Solińska J, Urbanowicz A, Figlerowicz M, Bujarski JJ. RNA-RNA recombination in plant virus replication and evolution. Annu Rev Phytopathol. 2011;49(1):415-43.

70. Pérez-Losada M, Arenas M, Galán JC, Palero F, González-Candelas F. Recombination in viruses: mechanisms, methods of study, and evolutionary consequences. Infect Genet Evol. 2015;30:296-307.

71. Gough RE, Collins MS, Borland E, Keymer LF. Astrovirus-like particles associated with hepatitis in ducklings. Vet Rec. 1984;114(11):279.

72. Berger PH. Potyviridae. In: Fauquet CM, Mayo MA, Maniloff J, Desselberger U, Ball LA, editors. Virus Taxonomy. San Diego: Academic; 2005. p. 819-41.

73. Benson DA, Clark K, Karsch-Mizrachi I, Lipman DJ, Ostell J, Sayers EW. GenBank. Nucleic Acids Res. 2015;43(D1):D30-5.

74. Kearse M, Moir R, Wilson A, Stones-Havas S, Cheung M, Sturrock S, Buxton S, Cooper A, Markowitz S, Duran C, et al. Geneious Basic: an integrated and extendable desktop software platform for the organization and analysis of sequence data. Bioinformatics. 2012;28(12):1647-9.

75. Bi S-L, Purdy MA, McCaustland KA, Margolis HS, Bradley DW. The sequence of hepatitis $E$ virus isolated directly from a single source during an outbreak in China. Virus Res. 1993;28(3):233-47.

76. Altschul SF, Gish W, Miller W, Myers EW, Lipman DJ. Basic local alignment search tool. J Mol Biol. 1990;215(3):403-10.

77. Altschul SF, Madden TL, Schaffer AA, Zhang J, Zhang Z, Miller W, Lipman DJ. Gapped BLAST and PSI-BLAST: a new generation of protein database search programs. Nucleic Acids Res. 1997;25(17):3389-402.

78. Boratyn GM, Schaffer AA, Agarwala R, Altschul SF, Lipman DJ, Madden TL. Domain enhanced lookup time accelerated BLAST. Biol Direct. 2012;7:12.

79. Jones P, Binns D, Chang H-Y, Fraser M, Li W, McAnulla C, McWilliam H, Maslen J, Mitchell A, Nuka G, et al. InterProScan 5: genome-scale protein function classification. Bioinformatics. 2014;30(9):1236-40.

80. Mitchell A, Chang H-Y, Daugherty L, Fraser M, Hunter S, Lopez R, McAnulla C, McMenamin C, Nuka G, Pesseat S, et al. The InterPro protein families database: the classification resource after 15 years. Nucleic Acids Res. 2015; 43(D1):D213-21.

81. Katoh K, Kuma K-i, Toh H, Miyata T. MAFFT version 5: improvement in accuracy of multiple sequence alignment. Nucleic Acids Res. 2005;33(2):511-8.

82. Darriba D, Taboada GL, Doallo R, Posada D. ProtTest 3: fast selection of bestfit models of protein evolution. Bioinformatics. 2011;27(8):1164-5.

83. Guindon S, Gascuel O. A simple, fast, and accurate algorithm to estimate large phylogenies by maximum likelihood. Syst Biol. 2003;52(5):696-704.

84. Le SQ, Gascuel O. An Improved General Amino Acid Replacement Matrix. Mol Biol Evol. 2008;25(7):1307-20.

85. Müller T, Vingron M. Modeling amino acid replacement. J Comput Biol. 2000;7(6):761-76.

86. Balayan MS, Andjaparidze AG, Savinskaya SS, Ketiladze ES, Braginsky DM, Savinov AP, Poleschuk VF. Evidence for a virus in non-A, non-B hepatitis transmitted via the fecal-oral route. Intervirology. 1983;20(1):23-31.

87. Tam AW, Smith MM, Guerra ME, Huang C-C, Bradley DW, Fry KE, Reyes GR. Hepatitis E virus (HEV): Molecular cloning and sequencing of the full-length viral genome. Virology. 1991;185(1):120-31.

88. Reyes GR, Purdy MA, Kim JP, Luk KC, Young LM, Fry KE, Bradley DW. Isolation of a cDNA from the virus responsible for enterically transmitted non-A, non-B hepatitis. Science. 1990;247(4948):1335-9.

\section{Submit your next manuscript to BioMed Central and we will help you at every step:}

- We accept pre-submission inquiries

- Our selector tool helps you to find the most relevant journal

- We provide round the clock customer support

- Convenient online submission

- Thorough peer review

- Inclusion in PubMed and all major indexing services

- Maximum visibility for your research

Submit your manuscript at www.biomedcentral.com/submit
Biomed Central 